\title{
No slow-roll inflation à la Generalized Chaplygin Gas in General Relativity
}

\author{
Alexander Gallego Cadavid, J.R. Villanueva \\ Instituto de Física y Astronomía, Universidad de Valparaíso, \\ Avenida Gran Bretaña 1111, Playa Ancha, Valparaíso, Chile
}

The Generalized Chaplygin Gas (GCG) model is characterized by the equation of state $P=-A \rho^{-\alpha}$, where $A>0$ and $\alpha<1$. The model has been extensively studied due to its interesting properties and applicability in several contexts, from late-time acceleration to primordial inflation. Nonetheless we show that the inflationary slow-roll regime cannot be satisfied by most of the parameter space of the GCG model when General Relativity (GR) is considered. In particular, although the model has been applied to inflation with $0<\alpha<1$, we show that for $-1<\alpha \leq 1$ there is no expansion of the Universe but an accelerated contraction. For $\alpha \leq-5 / 3$, the second slow-roll parameter $\eta_{H}$ is larger than unity, so there is no sustained period of inflation. Only for $\alpha$ very close to -1 the model produces enough $e$-folds, thus greatly reducing its parameter space. Moreover, we show that the model is ruled out by the Planck 2018 results. Finally, we extend our analysis to the Generalized Chaplygin-Jacobi Gas (GCJG) model. We find that the introduction of a new parameter does not change the previous results. We thus conclude that the violation of the slow-roll conditions is a generic feature of the GCG and GCJG models during inflation when GR is considered and that the models are ruled out by the Planck 2018 results.

\section{INTRODUCTION}

An important prediction of inflationary cosmology is that there should be small departures from the large-scale homogeneity observed in the Universe [1, 2]. Inflation predicts that these perturbations have a characteristic spectrum. During inflation, space-time itself fluctuates quantum mechanically about a background in an accelerated expanding phase [1-9]. These quantum fluctuations are the seeds for the observed structures and CMB anisotropies since the microscopic fluctuations were spread out to macroscopic scales, where they eventually become classical fluctuations in space-time [1, 2, 6, 9].

The simplest models of cosmic inflation involve a scalar field $\phi$, called the inflaton, slowly rolling down a very flat energy potential $V(\phi)[1]$. During inflation most of the Universe's energy density is in the form of $V(\phi)$. In these circumstances the field behaves like a fluid with negative pressure and thus powers an almost exponential cosmic expansion. Inflation ends when the inflaton's kinetic energy is larger than its potential energy, which occurs in the steeper part of $V(\phi)$. Nonetheless, 
before the end of inflation, it is crucial to have enough slow rolling for the scalar field in order to solve the horizon and flatness problems with inflation [1, 2, 6, 9]. This corresponds to a growing of the scale factor $a$ of the order of $e^{60}[1-10]$.

Recently, the generalized Chaplygin gas (GCG) model has been discussed widely in cosmological contexts. The model is characterized by a fluid with an exotic equation of state

$$
P=-\frac{A}{\rho^{\alpha}}
$$

where $A>0$ and $\alpha<1$ [11 14]. The model corresponds to a generalized Nambu-Goto action which can be interpreted as a perturbed $d$-brane in a $(d+1,1)$ spacetime [11]. The case $\alpha=1$ reproduces the pure Chaplygin gas model in Ref. [15], while $\alpha=-1$ corresponds to a cosmological constant [11 20]. In the late-time cosmological context and for $\alpha<-1$, Eq. (1) leads to three new versions of the GCG model: an early phantom model, a late phantom model, and a transient model [12].

Originally, the Chaplygin gas model was introduced to explain late-time acceleration without dark energy in the context of General Relativity (GR) [11 20]. So far there have been different modifications to the GCG proposal such as modified Chaplygin gas in brane-world [21 23] and GCG in modified gravity [14, 24, 25].

A first approach to single-field inflation using the GCG as the inflaton field was done in Ref. [26] using $0<\alpha<1$, where the value $\alpha=0.2578 \pm 0.0009$ was found from the Planck 2013 data. Later the GCG inflation was studied in light of Planck where it was shown that the GCG is not a suitable candidate for inflation in the context of GR for $\alpha<-1$ [13]. In this work the authors found that, in order to obtain the observational Planck+ WP and BICEP2 bounds for the spectral index and the tensor-to-scalar ratio, the number of $e$-folds before the end of inflation at horizon exit $N_{*}$ for the modes would have to be of order $N_{*} \approx 217$, which is way out of the theoretical bound $50<N_{*}<60$ [1, 2]. More recently, a further generalization of the GCG model was studied using elliptic functions to describe the inflationary epoch [27, 28]. There the inflaton field was characterized by an equation of state corresponding to a generalized Chaplygin-Jacobi gas (GCJG)

$$
P=-\frac{A \kappa}{\rho^{\alpha}}-2(1-\kappa) \rho+\frac{(1-\kappa)}{A} \rho^{2+\alpha}
$$

where $\alpha$ is the GCG parameter, $0 \leq \alpha \leq 1$, and $0 \leq \kappa \leq 1$ is the modulus of the elliptic function [27, 28].

In this paper we show that the GCG and GCJG models applied to inflation in Refs. [26] and [27, 28, in Eqs. (1) and (2) respectively, do not produce an inflationary epoch for the values of the parameters studied there. We then extend the $\alpha$-parameter space to $\alpha<0$ for the models and 
show that there is a sustained inflationary period only for $\alpha$ very close to -1 . Thus, the parameter space for the models is greatly reduced.

The layout of the paper is the following. In Sections II and III we review single-field inflation and the Hamilton-Jacobi formalism, respectively. There we emphasis on the number of $e$-folds during inflation and the slow-roll conditions. In Section IV we apply the Hamilton-Jacobi formalism in order to study primordial inflation using a fluid which presents the properties of the GCG. Then in Section $\mathrm{V}$ we show that for $\alpha>-1$ the GCG model does not produce an inflationary epoch. In Section $\mathrm{VI}$ we show that the GCG model produces a burst of inflation only for $\alpha>-5 / 3$, since the slow-roll conditions are not satisfied for $\alpha \leq-5 / 3$. We also constrain the model from the Planck 2018 results. In Section VII we extend our analysis to the case of the GCJG where we find similar results. The conclusions are presented in Section VIII. In this paper we will restrict ourselves to the study of cosmic inflation using the GCG and GCJG models in the context of GR. Throughout this work we use natural units $\left(c=\hbar=k_{B}=1\right)$.

\section{SINGLE-FIELD INFLATION}

Cosmic inflation is a period of accelerated expansion in the early Universe where the scale factor $a$ behaves like [1 9 ]

$$
\ddot{a}>0 \text {. }
$$

In the simplest models, inflation is driven by the canonical inflaton $\phi$, slowly rolling the smooth potential energy $V(\phi)$. Throughout this paper we consider a Friedmann-Lemaître-RobertsonWalker (FLRW) metric in a flat Universe

$$
\mathrm{d} s^{2}=-\mathrm{d} t^{2}+a^{2}(t) \mathrm{d} \vec{x}^{2}=a^{2}(\tau)\left[-\mathrm{d} \tau^{2}+\mathrm{d} \vec{x}^{2}\right]
$$

where $d \tau \equiv d t / a$ is the conformal time and $\mathrm{d} \vec{x}^{2}$ is the metric on a maximally symmetric $3-$ manifold. We assume that the stress energy of the Universe is dominated by the inflaton $\phi$, such that the Einstein field equations of the background metric are given by [6, 8,9$]$

$$
H^{2} \equiv\left(\frac{\dot{a}}{a}\right)^{2}=\frac{8 \pi}{3 m_{\mathrm{Pl}}^{2}}\left[V(\phi)+\frac{1}{2} \dot{\phi}^{2}\right],
$$

and

$$
\left(\frac{\ddot{a}}{a}\right)=\frac{8 \pi}{3 m_{\mathrm{Pl}}^{2}}\left[V(\phi)-\dot{\phi}^{2}\right],
$$


where $H$ is the Hubble parameter, $m_{\mathrm{Pl}} \equiv G^{-1 / 2}$ is the Planck mass, and dots denote derivatives with respect to cosmic time.

The equation of motion of the spatially homogeneous scalar field is given by [6, 8 , 9 ]

$$
\ddot{\phi}+3 H \dot{\phi}+V^{\prime}(\phi)=0
$$

where primes denote derivatives with respect to $\phi$.

\section{Number of $e$-folds and the slow-roll parameters}

The amount of inflation is quantified by the ratio of the scale factor at the final time to its value at some initial time $t_{i}$. This ratio is normally a large number thus it is customary to take the logarithm to give the number of $e$-folds

$$
N(t) \equiv \ln \left[\frac{a(t)}{a\left(t_{i}\right)}\right]=\int_{a_{i}}^{a} \frac{d \tilde{a}}{\tilde{a}}=\int_{t_{i}}^{t} H(\tilde{t}) d \tilde{t} .
$$

In order to obtain at least $N \sim 60$ we need to impose that $H$ does not change much within a Hubble time $H^{-1}$, i.e., $d H^{-1} / d t \ll 1[9]$. This requisite is equivalent to the first slow-roll condition

$$
\epsilon \equiv-\frac{\dot{H}}{H^{2}} \ll 1
$$

The second slow-roll condition is given by the requirement that $\epsilon$ does not change much within a Hubble time

$$
\eta \equiv \frac{\dot{\epsilon}}{\epsilon H} \ll 1
$$

The slow-roll approximation applies when these parameters are small in comparison to unity, i.e., $\epsilon,|\eta| \ll 1$. As long as $\epsilon<1$, a successful period of inflation can be realized even when $|\eta|>1$ for a few $e$-folds 29 31. On the other hand the violation of the second slow-roll condition, i.e. $|\eta|>1$, implies that $\epsilon$ grows very rapidly bringing inflation to a swifter end, and thus yielding a small number of $e$-folds [31]. As we will see below, this aspect is crucial in our study of the GCG and GCJG models during inflation since we will prove below that, generically, $|\eta|>1$. Hence the GCG and GCJG models produce a small number of $e$-folds.

\section{THE HAMILTON-JACOBI FORMALISM OF SINGLE-FIELD INFLATION}

In general, the Hubble parameter $H$ will vary as the inflaton field $\phi$ evolves along the potential energy $V(\phi)$. In some cases a more convenient approach is to express the Hubble parameter 
directly as a function of the field $\phi$ instead of as a function of time, i.e., $H=H(\phi)$. In this section we will follow this path, known as the Hamilton-Jacobi formalism [13, 31 33.

We start by differentiating Eq. (5) with respect to time from which we obtain [13]

$$
2 H(\phi) H^{\prime}(\phi) \dot{\phi}=-\left(\frac{8 \pi}{m_{\mathrm{Pl}}^{2}}\right) H(\phi) \dot{\phi}^{2},
$$

and where we used Eq. (7) to eliminate $\ddot{\phi}$. Substituting back into the definition of $H$ in Eq. (5) results in the system of two first-order equations

$$
\begin{aligned}
\dot{\phi} & =-\frac{m_{\mathrm{Pl}}^{2}}{4 \pi} H^{\prime}(\phi), \\
{\left[H^{\prime}(\phi)\right]^{2}-\frac{12 \pi}{m_{\mathrm{Pl}}^{2}} H^{2}(\phi) } & =-\frac{32 \pi^{2}}{m_{\mathrm{Pl}}^{4}} V(\phi) .
\end{aligned}
$$

These equations are completely equivalent to the second-order equation of motion in Eq. (7). The second of these is referred to as the Hamilton-Jacobi equation [13, 31,33]. It allows us to consider $H(\phi)$ as the fundamental quantity to be specified, instead of the usual potential energy $V(\phi)$. In cases where $H(\phi)$ is known, the Hamilton-Jacobi approach is very useful in obtaining several inflationary quantities. For instance, from Eq. 12 we may obtain an explicit expression for the inflaton field in terms of the cosmological time $t^{1}$. From Eq. 13 the inflaton potential is given by

$$
V(\phi)=\left(\frac{3 m_{\mathrm{Pl}}^{2}}{8 \pi}\right)\left[H^{2}(\phi)-\frac{m_{\mathrm{Pl}}^{2}}{12 \pi}\left[H^{\prime}(\phi)\right]^{2}\right] \text {. }
$$

Moreover, by multiplying Eq. 12 by $d a / d \phi$ we can also obtain an expression for the scale factor in the form

$$
a(\phi)=a_{i} \exp \left\{-\frac{4 \pi}{m_{\mathrm{Pl}}^{2}} \int_{\phi_{i}}^{\phi} \frac{H(\tilde{\phi})}{H^{\prime}(\tilde{\phi})} d \tilde{\phi}\right\} .
$$

Finally, from this last expression, assuming that we have the scalar field as a function of time, we can obtain the scale factor as a function of cosmological time.

In this formalism the slow-roll parameters are defined as [13, $31+33$

$$
\begin{aligned}
\epsilon_{H}(\phi) & \equiv \frac{m_{\mathrm{Pl}}^{2}}{4 \pi}\left(\frac{H^{\prime}(\phi)}{H(\phi)}\right)^{2}, \\
\eta_{H}(\phi) & \equiv \frac{m_{\mathrm{Pl}}^{2}}{4 \pi} \frac{H^{\prime \prime}(\phi)}{H(\phi)}, \\
\xi_{H}^{2}(\phi) & \equiv\left(\frac{m_{\mathrm{Pl}}^{2}}{4 \pi}\right)^{2} \frac{H^{\prime}(\phi) H^{\prime \prime \prime}(\phi)}{H^{2}(\phi)},
\end{aligned}
$$

\footnotetext{
${ }^{1}$ This is consistent as long as $t$ is a single-valued function of $\phi$.
} 
where we have introduced a third $\xi_{H}$ slow-roll parameter which is important in the study of scalar perturbations.

The inflationary condition in Eq. (3), $\ddot{a}>0$, is precisely equivalent to the condition $\epsilon_{H}<1$. In order to see this Eq. (6) can be written as

$$
\left(\begin{array}{l}
\ddot{a} \\
a
\end{array}\right)=H^{2}(\phi)\left[1-\epsilon_{H}(\phi)\right]
$$

such that inflation ends once $\epsilon_{H}$ exceeds unity. Note that the conditions leading to a violation of the strong energy condition are uniquely determined by the magnitude of $\epsilon_{H}$ alone [31. As we stated before, inflation can still proceed if $\left|\eta_{H}\right|$ or $\left|\xi_{H}\right|$ are much larger than unity, though normally such values would drive a rapid variation of $\epsilon_{H}$ and bring about a swift end to inflation [31 40].

So far we have reviewed cosmic inflation and the Hamilton-Jacobi formalism. In the next sections we will study the GCG model in the Hamilton-Jacobi approach. In Section $[\mathrm{V}$ we will use this formalism to show that, in contradiction to previous results [26] 28], inflation does not proceed when $\alpha>-1$ for the the GCG model. Then in Section VI we prove that the slow-roll condition $\left|\eta_{H}\right|<1$ only holds for $-5 / 3<\alpha<-1$.

\section{INFLATION À LA GENERALIZED CHAPLYGIN GAS}

In this section we apply the Hamilton-Jacobi formalism in order to study primordial inflation using a fluid which presents the properties of a GCG with equation of state given by Eq. (1).

In this formalism the generating function $H(\phi)$ is given by [13, 26]

$$
H(\phi)=H_{0} \cosh ^{\frac{1}{1+\alpha}}\left[\sqrt{\frac{6 \pi}{m_{\mathrm{Pl}}^{2}}}(1+\alpha)\left(\phi-\phi_{0}\right)\right],
$$

where

$$
H_{0}=H\left(\phi_{0}\right) \equiv \sqrt{\frac{8 \pi}{3 m_{\mathrm{Pl}}^{2}}} A^{\frac{1}{2(1+\alpha)}},
$$

and $\phi_{0}$ is an integration constant given by

$$
\phi_{0}=\phi_{i}-\frac{1}{(1+\alpha)} \sqrt{\frac{m_{\mathrm{Pl}}^{2}}{6 \pi}} \operatorname{arcsinh}\left[\sqrt{\frac{\rho_{i}^{1+\alpha}}{A}-1}\right],
$$

where $\rho_{i}=\rho\left(\phi_{i}\right)$ is the energy density at the beginning of inflation and $\rho_{i}^{1+\alpha} / A>1$ [13]. The condition $\rho_{i}^{1+\alpha} / A>1$ comes from both the null energy condition $\rho+P \geq 0$ and the continuity equation $\dot{\rho}+3 \dot{a}(\rho+P) / a=0$. In the latter case, there is no solution to the continuity equation when $A=\rho_{i}^{1+\alpha}[13]$. 
Now using Eq. (15) we calculate the scale factor

$$
a(\phi)=a_{i} \exp \left\{-\frac{2}{3(1+\alpha)} \int_{\Phi_{i}}^{\Phi} \operatorname{coth}[(1+\alpha) \tilde{\Phi}] d((1+\alpha) \tilde{\Phi})\right\}=a_{i}\left(\frac{\sinh [(1+\alpha) \Phi]}{\sinh \left[(1+\alpha) \Phi_{i}\right]}\right)^{\frac{-2}{3(1+\alpha)}}
$$

where for simplicity we use the dimensionless variable

$$
\Phi(\phi) \equiv \sqrt{\frac{6 \pi}{m_{\mathrm{Pl}}^{2}}}\left(\phi-\phi_{0}\right)
$$

such that

$$
\Phi_{i}(\phi) \equiv \sqrt{\frac{6 \pi}{m_{\mathrm{Pl}}^{2}}}\left(\phi_{i}-\phi_{0}\right)=\frac{1}{1+\alpha} \operatorname{arcsinh}\left[\sqrt{\frac{\rho_{i}^{1+\alpha}}{A}-1}\right] .
$$

In Ref. [13] the authors calculated the same expression for $a(\phi)$ from the continuity equation.

The slow-roll parameters in Eqs. (16) - 18) are written as [26]

$$
\begin{aligned}
\epsilon_{H}(\phi) & =\frac{3}{2} \tanh ^{2}[(1+\alpha) \Phi], \\
\eta_{H}(\phi) & =\frac{3}{2}\left(1+\alpha \operatorname{sech}^{2}[(1+\alpha) \Phi]\right), \\
\xi_{H}^{2}(\phi) & =\frac{9}{8}\left(1-2 \alpha-4 \alpha^{2}+\cosh [2(1+\alpha) \Phi]\right) \operatorname{sech}^{2}[(1+\alpha) \Phi] \tanh ^{2}[(1+\alpha) \Phi] .
\end{aligned}
$$

The condition for the end of inflation, $\epsilon_{H}\left(\Phi_{e}\right)=1$, yields

$$
\Phi_{e}(\alpha)=\frac{1}{1+\alpha} \operatorname{arctanh}\left(\sqrt{\frac{2}{3}}\right)
$$

or equivalently

$$
\phi_{e}(\alpha, A)=\phi_{0}+\sqrt{\frac{m_{\mathrm{Pl}}^{2}}{6 \pi}} \frac{1}{(1+\alpha)} \operatorname{arctanh}\left(\sqrt{\frac{2}{3}}\right) .
$$

Finally, the number of $e$-folds $N$ from the beginning of inflation in Eq. (8) is given by

$$
N(\Phi)=-\frac{2}{3(1+\alpha)} \ln \left\{\frac{\sinh [(1+\alpha) \Phi]}{\sinh \left[(1+\alpha) \Phi_{i}\right]}\right\}
$$

thus that the total number of $e$-folds at the end of inflation $N_{e}$ is

$$
N_{e}(\alpha, A)=\frac{1}{3(1+\alpha)} \ln \left\{\frac{1}{2}\left(\frac{\rho_{i}^{1+\alpha}}{A}-1\right)\right\}
$$

where we use Eq. (22). As can be seen $N_{e}$ depends on the the GCG model parameters $\alpha$ and $A$ in such a way that for either $\alpha \rightarrow-1$ or $\rho_{i}^{1+\alpha} / A \rightarrow 1$, it can be arbitrarily large. We can also see that for $\rho_{i}^{1+\alpha} / A \geq 3$ the GCG model does not yield an inflationary period regardless of the value of $\alpha<-1$, since in this case $N \leq 0$. Hence $A$ is further constrained to $\rho_{i}^{1+\alpha}>A \geq \rho_{i}^{1+\alpha} / 3$. 

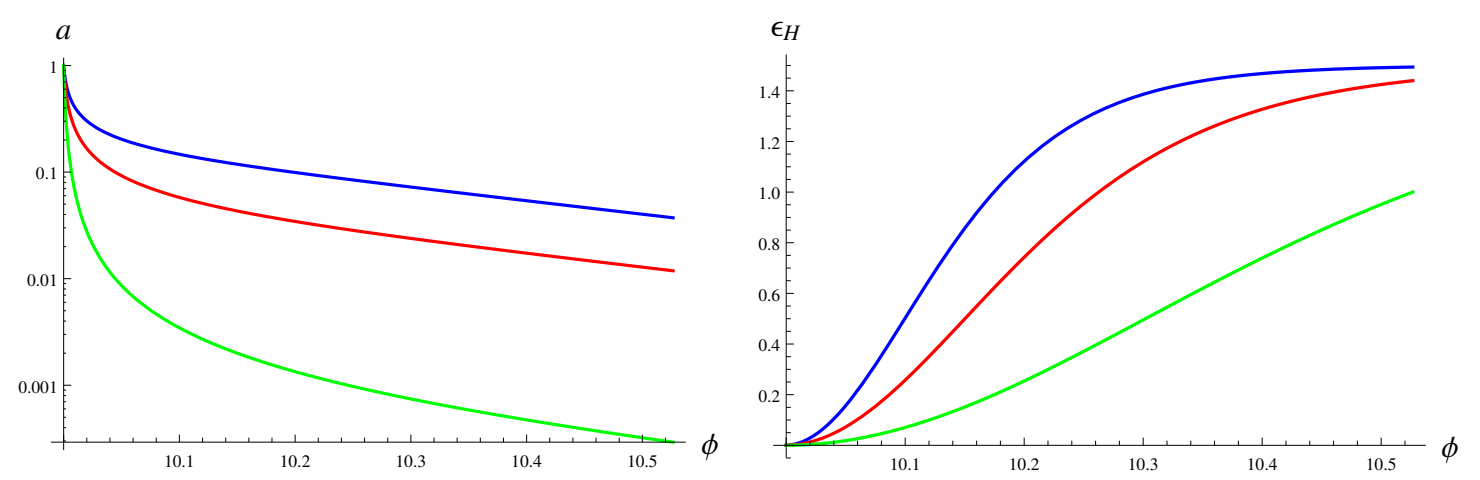

FIG. 1: The scale factor $a$ and the slow-roll parameter $\epsilon_{H}$ are plotted for $\alpha=0.5,0,-0.5$ and $\rho_{i}^{1+\alpha} / A=$ 1.00001 in the blue, red, and green lines, respectively. The other values chosen for the parameters are described at the end of Section IV] Notice that, although $\epsilon_{H}<1$, the scale factor is in fact decreasing. This can also be seen in Eq. (23) where the scale factor is a decreasing function for $\alpha>-1$

Unless stated otherwise, we use the following initial conditions in the plots below. Since from Planck 2018 [2] the upper bound on the Hubble parameter during inflation is $H_{*}<5.4 \times 10^{-6} m_{\mathrm{Pl}}$, we use for the initial energy density ${ }^{2} \rho_{i}=1.082 \times 10^{-14} m_{\mathrm{Pl}}^{4}$. For the rest of the parameters we use $a_{i}=1, \phi_{i}=10 m_{\mathrm{Pl}}, \alpha<1$, and $\rho_{i}^{1+\alpha} / A=1.00001$, where we use $\rho_{i}^{1+\alpha} / A$ instead of $A$ for simplicity.

\section{NO INFLATION À LA GENERALIZED CHAPLYGIN GAS $(\alpha>-1)$}

In Ref. [26] the authors studied the GCG model in the context of inflation using the interval $0<\alpha<1$. In this section we extend the study to $-1<\alpha<1$ and show that the GCG model does not produce an accelerated expansion but an accelerated contraction.

From Eq. 23) we can see that in fact the scale factor is a decreasing function of $\phi$ for $\alpha>-1$. This equation should be compared with Eq. (3.7) in Ref. [26], where the minus sign in the exponent is missing. This was probably the reason why the authors considered the GCG model for inflation in the interval $0<\alpha<1$. Moreover, in Ref. [26] only the deceleration parameter $q=-\ddot{a} a / \dot{a}^{2}$, or equivalently $\epsilon_{H}$, was plotted. Unfortunately this parameter alone does not give information of weather $a$ is increasing or decreasing.

In Fig. 1 the scale factor $a$ and the slow-roll parameter $\epsilon_{H}$ are plotted as functions of $\phi$. There it can be seen that $a$ decreases when $\alpha>-1$ although $\epsilon_{H}<1$. This means that in the GCG model the Universe is contracting in an accelerated fashion when $\alpha>-1$.

\footnotetext{
${ }^{2}$ See Refs. 34 40] for similar energy scales.
} 

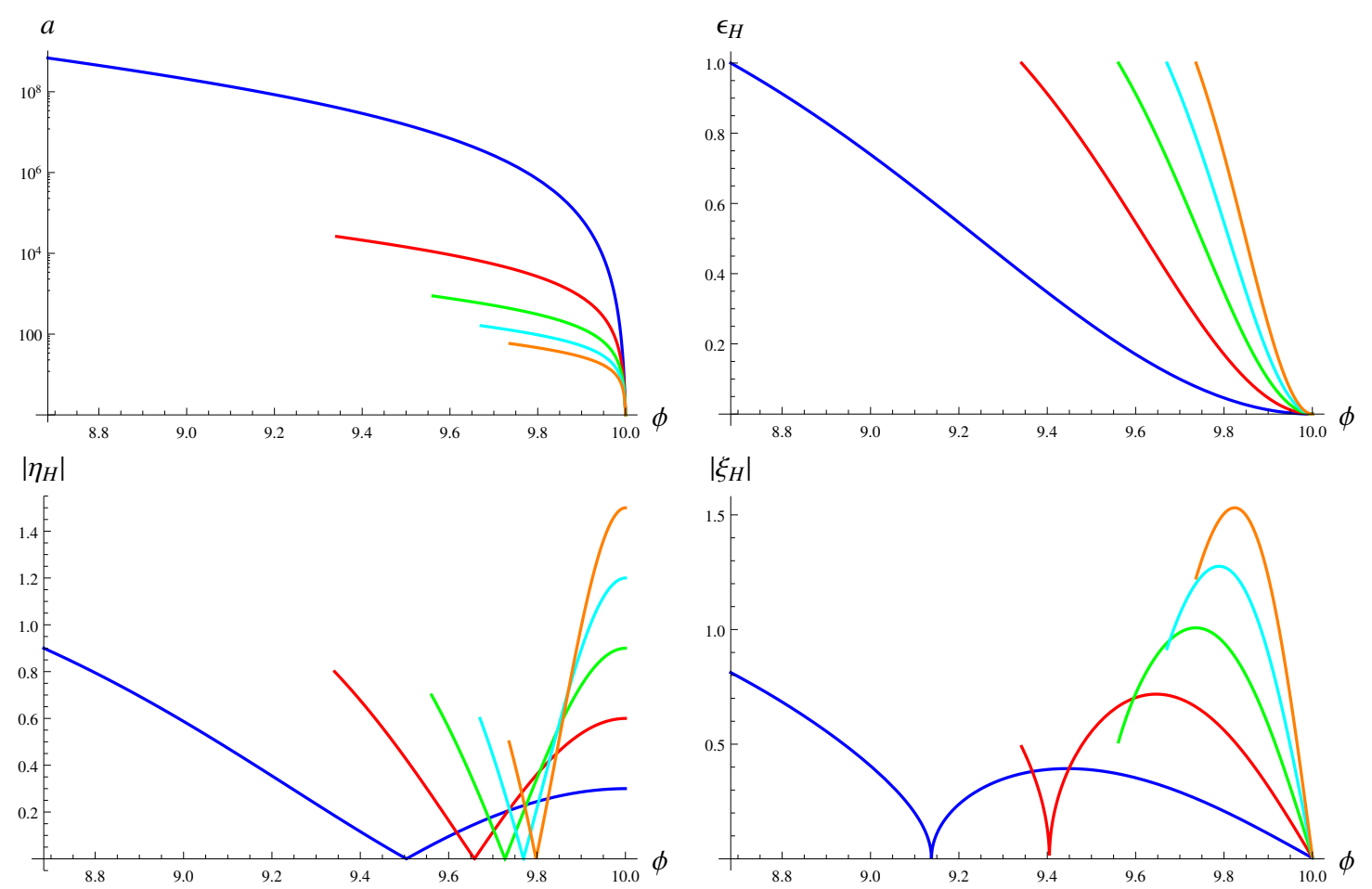

FIG. 2: The scale factor $a$ in Eq. 23 and the slow-roll parameters $\epsilon_{H}, \eta_{H}$ and $\xi_{H}^{2}$ in Eqs. 26 - 28 are plotted for several values of $\alpha$ and up to the end of inflation $\phi_{e}$ in Eq. (30). Here we choose $\alpha=$ $-1.2,-1.4,-1.6,-1.8,-2$, corresponding to the blue, red, green, cyan, and orange lines respectively. The other values chosen for the parameters are described at the end of Section IV] Here it can be seen that the conditions for a burst of inflation, $a$ increasing and $\left|\epsilon_{H}\right|<1$, are satisfied for $\alpha<-1$. It can also be seen that $\left|\eta_{H}\right|>1$ and $\left|\xi_{H}\right|>1$ for $\alpha \leq-5 / 3$ and $\alpha \leq-3 / 2$, respectively.

\section{NO SLOW-ROLL INFLATION À LA GENERALIZED CHAPLYGIN GAS $(\alpha<-1)$}

In this section we first show that the GCG model can indeed produce a burst of inflation when $\alpha<-1$. Then we prove that the slow-roll conditions $\left|\eta_{H}\right|<1$ and $\left|\xi_{H}\right|<1$ are only satisfied for the narrow range of values $-5 / 3<\alpha<-1$ and $-3 / 2<\alpha<-1$, respectively. Consequently we show that due to this behaviour of the slow-roll parameters we have that $N \ll 60$, thus the number of $e$-folds is not sufficient to solve the Big Bang problems during inflation unless $\alpha \rightarrow-1$.

In Fig. 2 we plot the scale factor $a$ and the slow-roll parameters $\epsilon_{H},\left|\eta_{H}\right|$, and $\left|\xi_{H}\right|$ for several values of $\alpha$ and up to the end of inflation $\phi_{e}$ (see Eq. (30)). As it can be seen the conditions for a burst of inflation are satisfied since $a$ is increasing and $\epsilon_{H}<1$ for all values of $\alpha<-1$. There it can also be seen that $\left|\eta_{H}\right|>1$ and $\left|\xi_{H}\right|>1$ for $\alpha \leq-5 / 3$ and $\alpha \leq-3 / 2$, respectively. This behaviour of $\eta_{H}$ and its effect on the number of $e$-folds will be studied below.

Let us find the values of $\alpha$ for which the GCG model fulfils $\left|\eta_{H}\right|<1$. In order to give an 


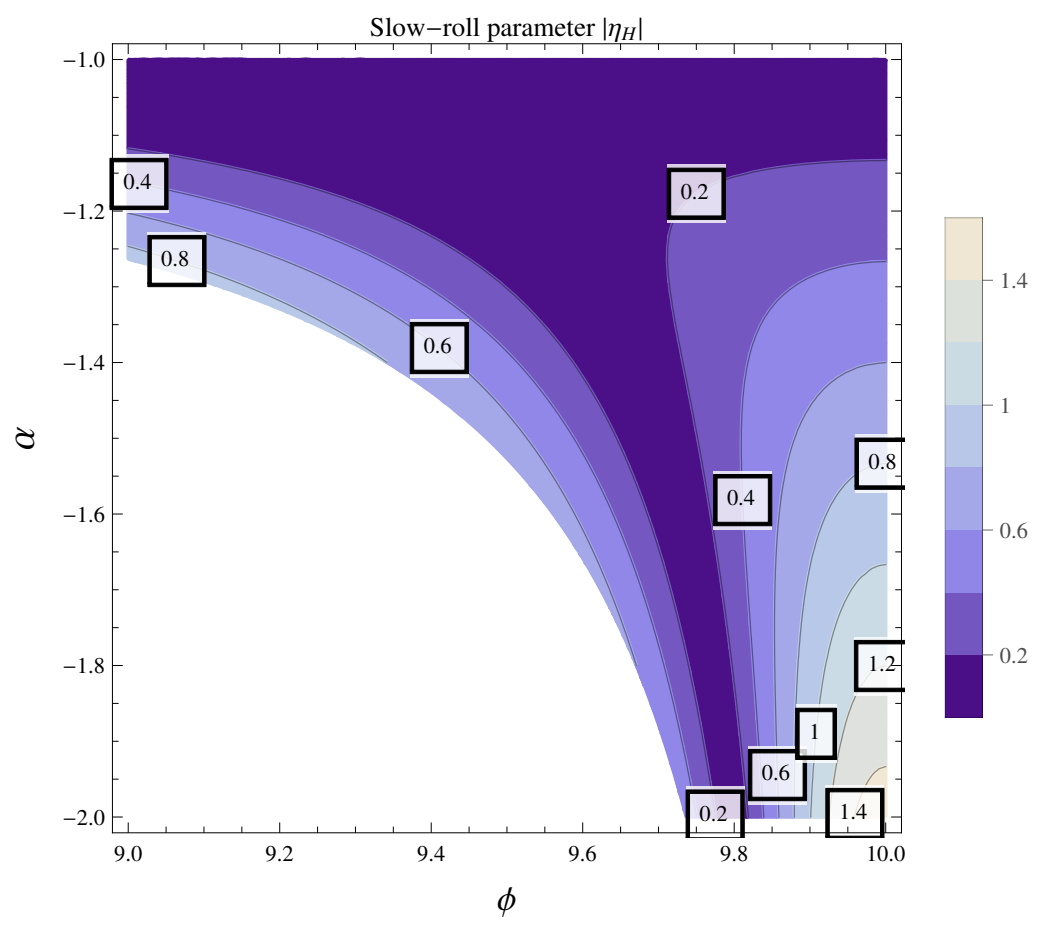

FIG. 3: The contour values of the slow-roll parameter $\eta_{H}$ are plotted as functions of $\phi \leq \phi_{e}$ (see Eq. (30)) and $-2<\alpha<-1$. Here it can be seen that $\left|\eta_{H}\right|>1$ for $\alpha \leq-5 / 3$ in agreement with our estimation in Eq. (34).

estimate, we consider $(1+\alpha) \Phi$ small. Then $\operatorname{sech}[(1+\alpha) \Phi] \sim 1$ such that $\left|\eta_{H}\right|<1$ in Eq. (27) becomes

$$
|1+\alpha|<\frac{2}{3}
$$

and, since we are only interested in $\alpha<-1$, we find that

$$
\left|\eta_{H}\right|<1 \quad \text { only for } \quad-\frac{5}{3}<\alpha<-1 \text {. }
$$

Similarly the condition for $\left|\xi_{H}\right|<1$, and assuming $(1+\alpha) \Phi$ small and $\Phi \sim 1$ such that $\tanh ^{2}[(1+\alpha) \Phi] \sim(1+\alpha)^{2}$, yields

$$
\left|\left(2-2 \alpha-4 \alpha^{2}\right)(1+\alpha)^{2}\right|<\frac{8}{9}
$$

from which we have

$$
\left|\xi_{H}\right|<1 \quad \text { only for } \quad-\frac{3}{2}<\alpha<-1
$$

In Figs. 3 and 4 we plot respectively the contour values of $\left|\eta_{H}\right|$ and $\left|\xi_{H}\right|$ as functions of $\phi$ and $\alpha$. In the plots it can be seen that $\left|\eta_{H}\right|>1$ and $\left|\xi_{H}\right|>1$ for $\alpha<-5 / 3$ and $\alpha<-1.6 \approx-3 / 2$, respectively, in agreement with our estimations. 


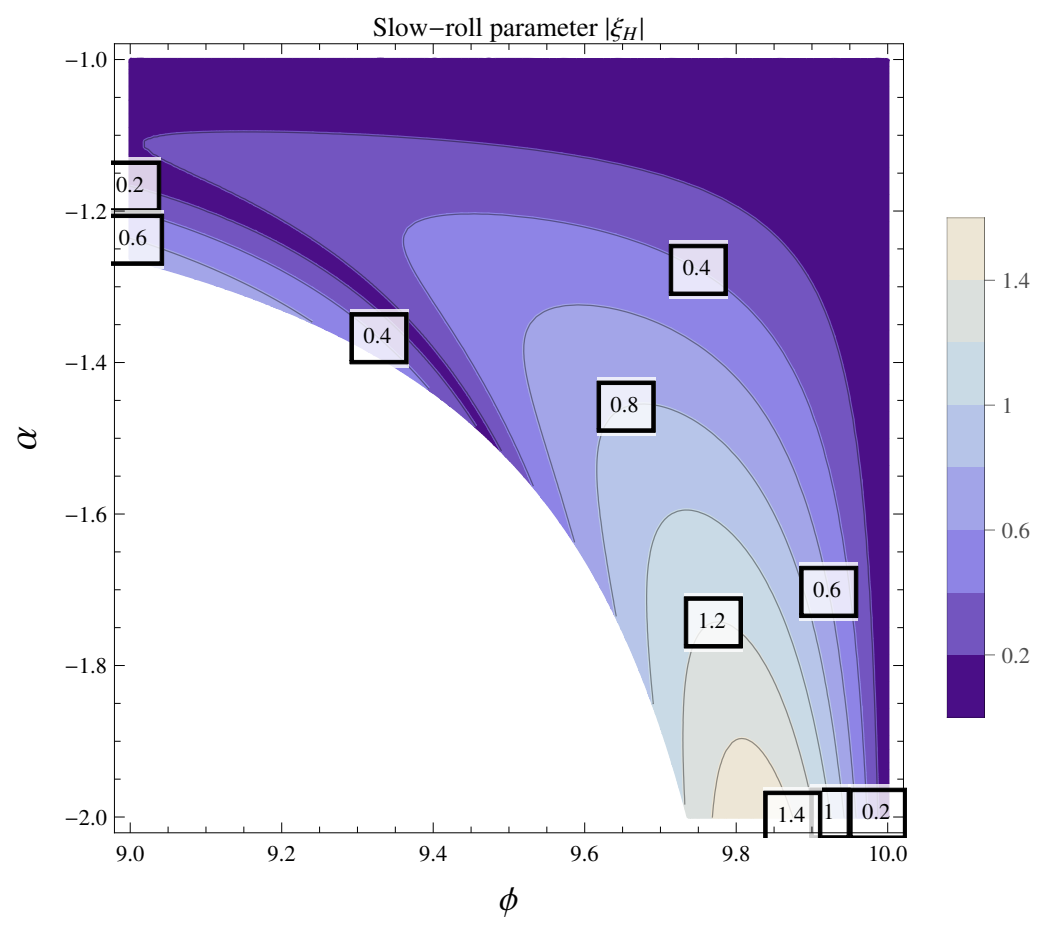

FIG. 4: The contour values of the slow-roll parameter $\left|\xi_{H}\right|$ are plotted as functions of $\phi \leq \phi_{e}$ (see Eq. (30)) and $-2<\alpha<-1$. Notice that $\left|\xi_{H}\right|$ is lager than unity for $\alpha<-1.6$ in relative agreement with our estimation in Eq. (36).
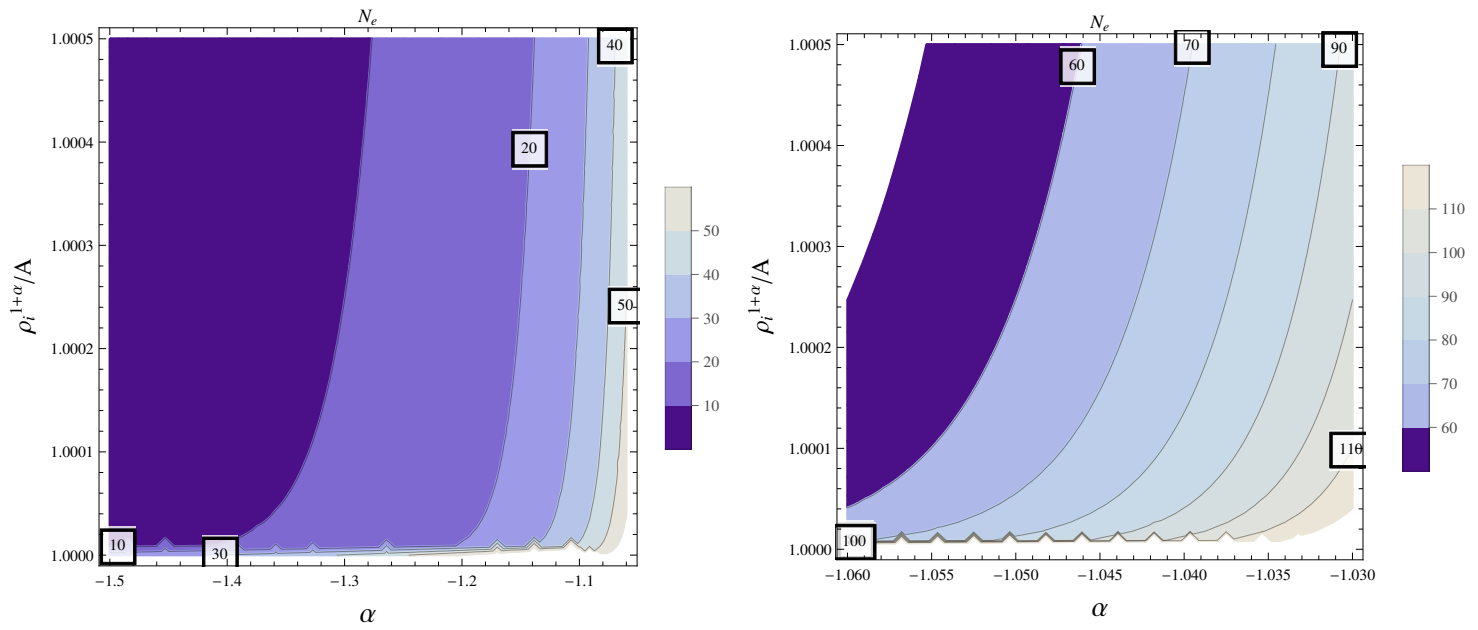

FIG. 5: The contour values of the number of $e$-folds at the end of inflation $N_{e}$ are plotted as functions of $\alpha$ and $\rho_{i}^{1+\alpha} / A$ (equivalently $A$ ) for the GCG model using Eq. 32. Here it can be seen that even for $\alpha<-1.1$ and $\rho_{i}^{1+\alpha} / A>1.0001$ we have $N<60$. The number of $e$-folds increases as either $\alpha \rightarrow-1$ or $\rho_{i}^{1+\alpha} / A \rightarrow 1$, although the effect $\alpha \rightarrow-1$ is larger. 
As anticipated, the behaviour of $\eta_{H}$ implies that the total number of $e$-folds $N$ would be too small for most of the parameter space of the GCG model. In Fig. 5 we plot the total number of $e$-folds at the end of inflation $N_{e}$ for the GCG model using Eq. (32). As it can be seen, in order to obtain at least $N_{e}>60$ either $\alpha \rightarrow-1$ or $\rho_{i}^{1+\alpha} / A \rightarrow 1$, although the effect $\alpha \rightarrow-1$ is larger. We recall that the case $\alpha=-1$ corresponds to a cosmological constant [11 20].

\section{Scalar and tensor perturbations}

We will now constrain the parameters of the GCG model from the study of the primordial perturbations. The evolution of the comoving curvature perturbation $\mathcal{R}$ is governed by the MukhanovSasaki equation [4]

$$
\frac{d^{2} u_{k}}{d \tau^{2}}+\left(k^{2}-\frac{1}{z} \frac{d^{2} z}{d \tau^{2}}\right) u_{k}=0
$$

where $k$ is the comoving wave number, $u \equiv-\operatorname{sign}(\dot{\phi}) z \mathcal{R} m_{\mathrm{Pl}} / \sqrt{8 \pi}$ is the Mukhanov-Sasaki variable, and $z=a \sqrt{2 \epsilon_{H}}$ such that

$$
\frac{1}{z} \frac{d^{2} z}{d \tau^{2}}=2 a^{2} H^{2}\left[1+f_{H}(\phi(\tau))\right]
$$

and where

$$
\begin{aligned}
f_{H}(\phi) & \equiv \epsilon_{H}-\frac{3}{2} \eta_{H}+\epsilon_{H}^{2}-2 \epsilon_{H} \eta_{H}+\frac{1}{2} \eta_{H}^{2}+\frac{1}{2} \xi_{H}^{2} \\
& =\frac{3}{8}\left(3(\alpha+1)(3 \alpha+2) \operatorname{sech}^{4}[(\alpha+1) \Phi]-(3 \alpha(2 \alpha+5)+7) \operatorname{sech}^{2}[(\alpha+1) \Phi]-2\right),
\end{aligned}
$$

where we use the slow-roll parameters in Eqs. (26) - 28) in the last line. Despite its appearance as an expansion in the slow-roll parameters, Eq. (38) is an exact expression. Here $f_{H}$ characterizes the validity of the slow-roll conditions, such that when $\left\{\epsilon_{H},\left|\eta_{H}\right|,\left|\xi_{H}\right|\right\}<1$ we have $\left|f_{H}\right| \ll 1$.

When the slow-roll regime is satisfied, $f_{H}$ can be neglected and the solution to the MukhanovSasaki equation can simply be written in terms of the Hankel function of the first kind $H_{\nu}(-k \tau)$. Nonetheless, as we have seen in the GCG model, the slow-roll approximations are only valid for $\alpha \rightarrow-1$ and $\rho_{i}^{1+\alpha} / A \rightarrow 1$. For instance, following a similar procedure as before we can estimate that

$$
\left|f_{H}\right|<1 \quad \text { only for } \quad-\frac{\sqrt{17}}{3}<\alpha<-1
$$

which is an even tighter constrain than the one for the slow-roll parameters separately. In Fig. 6 it can be seen that only for $\alpha \gtrsim-1.04$ the GCG model has $\left|f_{H}\right| \ll 1$ such that $f_{H}$ can be safely neglected in Eq. (38). 


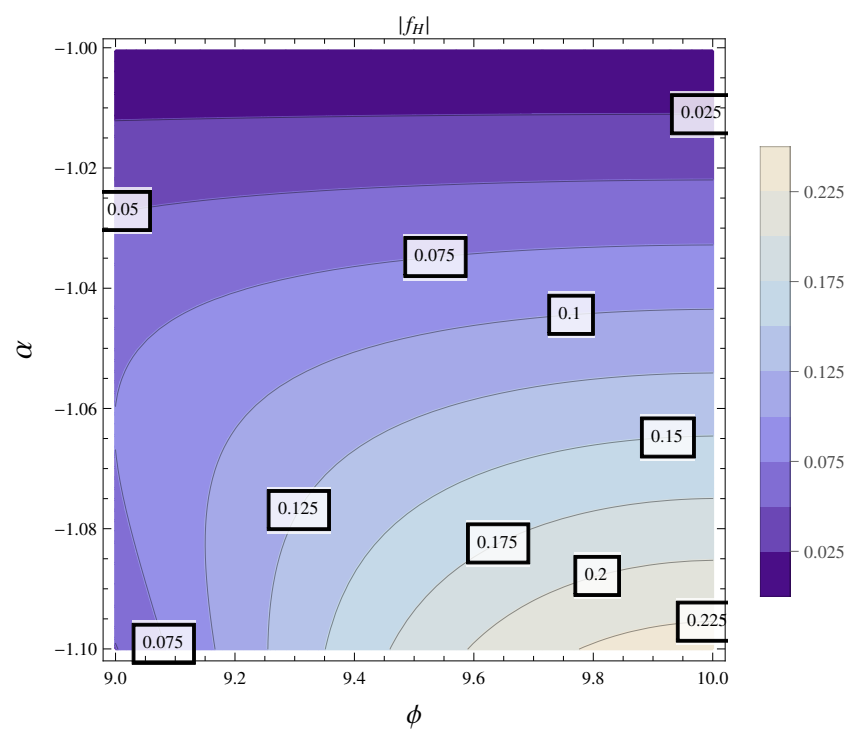

FIG. 6: The contour values of $\left|f_{H}\right|$ in Eq. (39) are plotted as functions of $\phi \leq \phi_{e}(\alpha, A)$ and $-1.1<\alpha<$ -1.0005 . As can be seen only for $\alpha \gtrsim-1.04$ the GCG model can indeed have $\left|f_{H}\right| \ll 1$ such that $f_{H}$ can be neglected in Eq. 38.

For the rest of this section we will only consider $-1.04<\alpha<-1$ such that the slow-roll approximation is valid. Under this approximation, the spectrum of comoving curvature perturbations $\mathcal{R}$ and the spectrum of tensor perturbations $h$ are given by [31]

$$
\begin{aligned}
P_{\mathcal{R}}\left(k_{*}\right) & =\left.\frac{1}{\pi m_{\mathrm{Pl}}^{2}} \frac{H^{2}}{\epsilon}\right|_{k_{*}=a_{*} H_{*}}, \\
P_{h}\left(k_{*}\right) & =\left.\frac{16}{\pi m_{\mathrm{Pl}}^{2}} H^{2}\right|_{k_{*}=a_{*} H_{*}},
\end{aligned}
$$

where the scalar and tensor power spectra are evaluated at the value of the inflaton field $\phi_{*}$ where the mode $k_{*}=a_{*} H_{*}$ crosses the Hubble radius for the first time [1, 2].

Following Ref. [2] and using Eq. (31), we can relate the number of e-folds $N_{*}$ before the end of inflation with the value of the field at horizon crossing $\phi_{*}$ by

$$
\phi_{*}=\phi_{0}+\sqrt{\frac{m_{\mathrm{Pl}}^{2}}{6 \pi}} \frac{1}{1+\alpha} \operatorname{arcsinh}\left[\sqrt{2 e^{3(1+\alpha) N_{*}}}\right] .
$$

The scalar spectral index $n_{s}$ and the tensor-to-scalar ratio $r$ are given by [31]

$$
\begin{aligned}
n_{s}-1 & \equiv \frac{d \ln P_{\mathcal{R}}}{d \ln k}=-4 \epsilon_{H}+2 \eta_{H}, \\
r & \equiv \frac{P_{h}}{P_{\mathcal{R}}}=16 \epsilon_{H},
\end{aligned}
$$

where again the quantities are evaluated at the horizon crossing scale $k_{*}=a_{*} H_{*}$. The Planck 


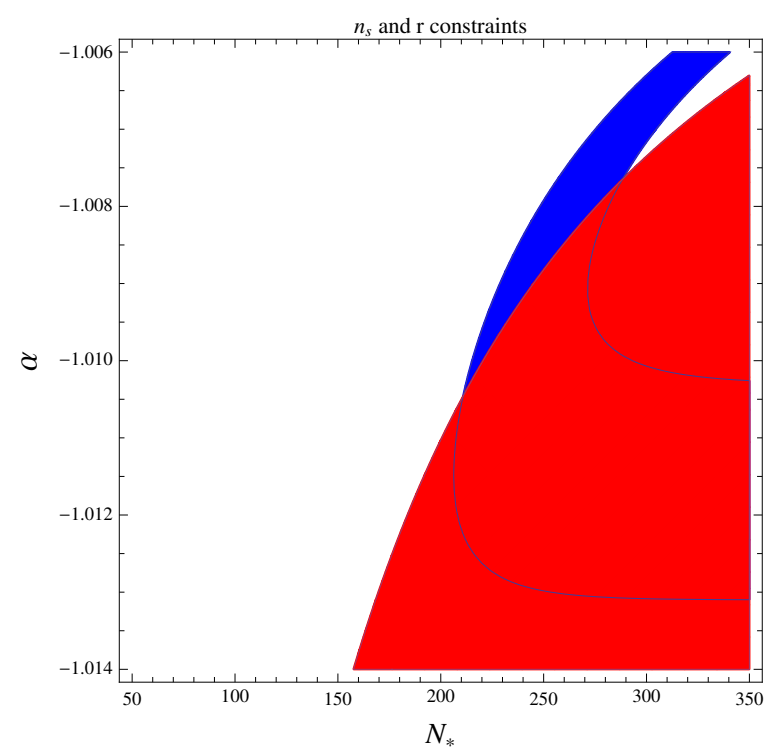

FIG. 7: The allowed region in the $\left(N_{*}, \alpha\right)$ plane. The blue (red) region corresponds to the values of $N_{*}$ and $\alpha$ for which the constraints from Planck 2018 on $n_{s}(r)$ are fulfilled. Here it can be seen that, in order to simultaneously fulfill the constraints, the number of $e$-folds before the end of inflation $N_{*}$ is way out the bounds $50<N_{*}<60$ [1, 2].

2018 bounds on the power spectrum amplitude, spectral index, and tensor-to-scalar ratio are, respectively, $A_{s}=(2.0989 \pm 0.10141) \times 10^{-9}, n_{s}=0.9649 \pm 0.0042$, and $r<0.064[1$, 2].

Using the expression for $\phi_{*}$ in Eq. (44), the scalar spectral index and tensor-to-scalar ratio at horizon crossing can be written, respectively, as

$$
\begin{aligned}
n_{s}\left(N_{*}, \alpha\right) & =-2+\frac{3(2+\alpha)}{1+2 e^{3(1+\alpha) N_{*}}}, \\
r\left(N_{*}, \alpha\right) & =\frac{24}{1+\frac{1}{2} e^{-3(1+\alpha) N_{*}}} .
\end{aligned}
$$

In Fig. 7 we plot the allowed region in the $\left(N_{*}, \alpha\right)$ plane satisfying the above Planck 2018 constraints. Since the relevant scales that can be probed through CMB observations are those that exited the horizon around $50<N_{*}<60$ [1, 2], it can be seen in Fig. 7 that the GCG model is ruled out by the Planck 2018 constraints. Notice that these results are independent of the parameter $A$, thus the constraints on $A$ from the Planck 2018 bounds are not relevant.

In this section we have seen that the slow-roll condition $\left|\eta_{H}\right|<1$ is not satisfied by the GCG model when $\alpha \leq-5 / 3$ and thus $N_{e}(\alpha, A) \ll 60$. In fact we have seen that only for $\alpha \rightarrow-1$ we have $N_{e}(\alpha, A)>60$. Moreover, from the Planck 2018 results we found that the model is ruled out. Our results differ from those in Ref. [13] since in there the authors used the BICEP2 constraint of $r=0.2_{-0.05}^{+0.07}$. 


\section{NO SLOW-ROLL INFLATION À LA GENERALIZED CHAPLYGIN-JACOBI GAS}

In this section we study the GCJG model in Eq. (2) and extend the analysis of Refs. [27, 28] from $0<\alpha<1$ to $\alpha<1$. Here we show that, even when the extra parameter $\kappa$ is added, the GCJG model does not produce inflation for $-1<\alpha \leq 1$ and that the slow-roll conditions for $\left|\eta_{H}\right|$ and $\left|\xi_{H}\right|$ are only satisfied in a narrow range of values of $\alpha$ very close to -1 .

In the GCJG the generating function in Eq. (20) is written as [27, 28]

$$
H(\phi, \kappa)=H_{0} \mathrm{nc}^{\frac{1}{1+\alpha}}[(1+\alpha) \Phi]
$$

where $\operatorname{nc}(x)=1 / \operatorname{cn}(x)$, and $\operatorname{cn}(x) \equiv \operatorname{cn}(x \mid \kappa)$ is the Jacobi elliptic cosine function, and $\kappa$ is the modulus. The generating function of the GCG in Eq. (20) is recovered when $\kappa=1$. Similarly as in the GCG model case the scale factor is now

$$
a(\phi)=a_{i}\left(\frac{\operatorname{sd}[(1+\alpha) \Phi]}{\operatorname{sd}\left[(1+\alpha) \Phi_{i}\right]}\right)^{\frac{-2}{3(1+\alpha)}},
$$

where $\operatorname{sd}(x) \equiv \operatorname{sd}(x \mid \kappa)=\operatorname{sn}(x) / \operatorname{dn}(x)$. Notice the minus sign in the exponent, which is also missing in Eq. (3.14) of Ref. [27]. In Fig. 8 we plot the scale factor as a function of $\phi$ which shows that $a$ is indeed decreasing for $-1<\alpha \leq 1$.

The slow-roll parameters are now given by

$$
\begin{aligned}
& \epsilon_{H}=\frac{3}{2} \frac{\operatorname{dn}^{2}[(1+\alpha) \Phi] \operatorname{sn}^{2}[(1+\alpha) \Phi]}{\operatorname{cn}^{2}[(1+\alpha) \Phi]} \\
& \eta_{H}=\epsilon_{H}\left\{\frac{1+\alpha \operatorname{cn}^{2}[(1+\alpha) \Phi]}{\operatorname{sn}^{2}[(1+\alpha) \Phi]}+\frac{(1+\alpha)(1-\kappa)}{\operatorname{dn}^{2}[(1+\alpha) \Phi]}\right\} \\
& \xi_{H}^{2}=\epsilon_{H}^{2}\left\{\frac{\left(2 \alpha^{2}+7 \alpha+6\right)(1-\kappa)}{\operatorname{sn}^{2}[(1+\alpha) \Phi] \operatorname{dn}^{2}[(1+\alpha) \Phi]}+\frac{3}{2 \epsilon_{H}}\left((2 \kappa-1)-\kappa \alpha(1+2 \alpha) \operatorname{cn}^{2}[(1+\alpha) \Phi]\right)\right\},
\end{aligned}
$$

where $\operatorname{sn}(x) \equiv \operatorname{sn}(x \mid \kappa)$ and $\operatorname{dn}(x) \equiv \operatorname{dn}(x \mid \kappa)$ are the Jacobi elliptic sine and delta functions, respectively. The condition for the end of inflation, $\epsilon_{H}\left(\Phi_{e}\right)=1$, now yields [27]

$$
\Phi_{e}(\alpha, \kappa)=\frac{1}{1+\alpha} F[\arcsin (\sqrt{y}), \kappa]
$$

where $F(\varphi, \kappa)$ is the normal elliptic integral of the first kind and $y=\left[5-(25-24 \kappa)^{1 / 2}\right] /(6 \kappa)$.

The number of $e$-folds is now given by [27]

$$
N(\phi)=-\frac{2}{3(1+\alpha)} \ln \left\{\frac{\operatorname{sd}[(1+\alpha) \Phi]}{\operatorname{sd}\left[(1+\alpha) \Phi_{i}\right]}\right\} .
$$

Then the total number of $e$-folds at the end of inflation is

$$
N_{e}(\alpha, A, \kappa)=\frac{1}{3(1+\alpha)} \ln \left\{\frac{1}{\mathcal{G}(\kappa)}\left(\frac{\rho_{i}^{1+\alpha}}{A}-1\right)\right\}
$$



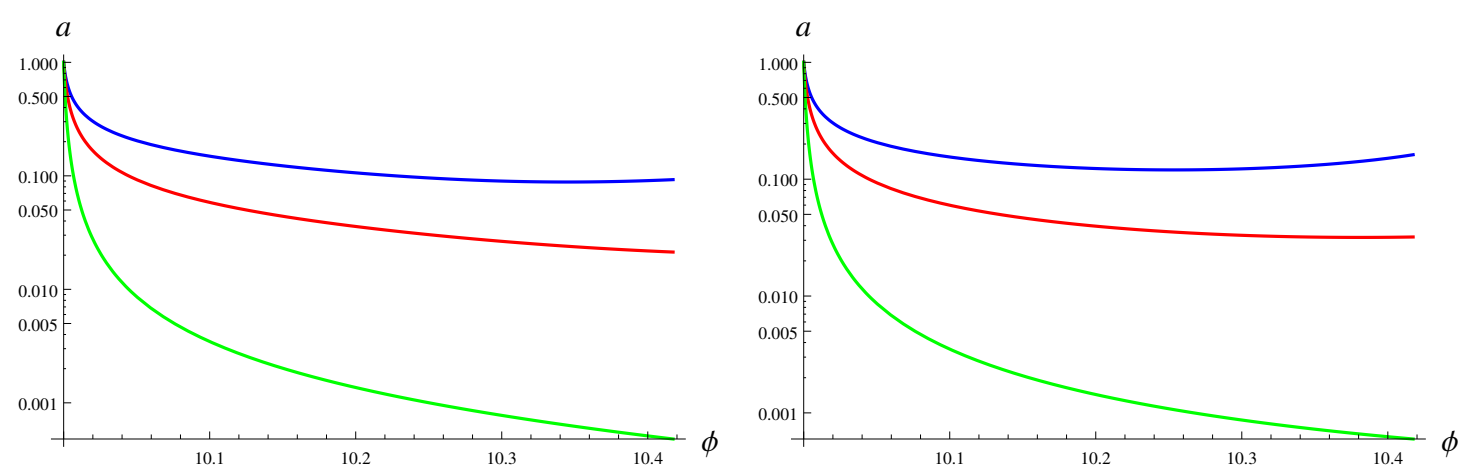

FIG. 8: The scale factor $a$ is plotted for $\alpha=0.5,0,-0.5$ and $\rho_{i}^{1+\alpha} / A=1.00001$ in the blue, red, and green lines, respectively. On the left (right) panel we use $\kappa=0.8(0.2)$. It can be seen that the scale factor is a decreasing function of $\phi$, hence there is no inflationary period for $\alpha>-1$.
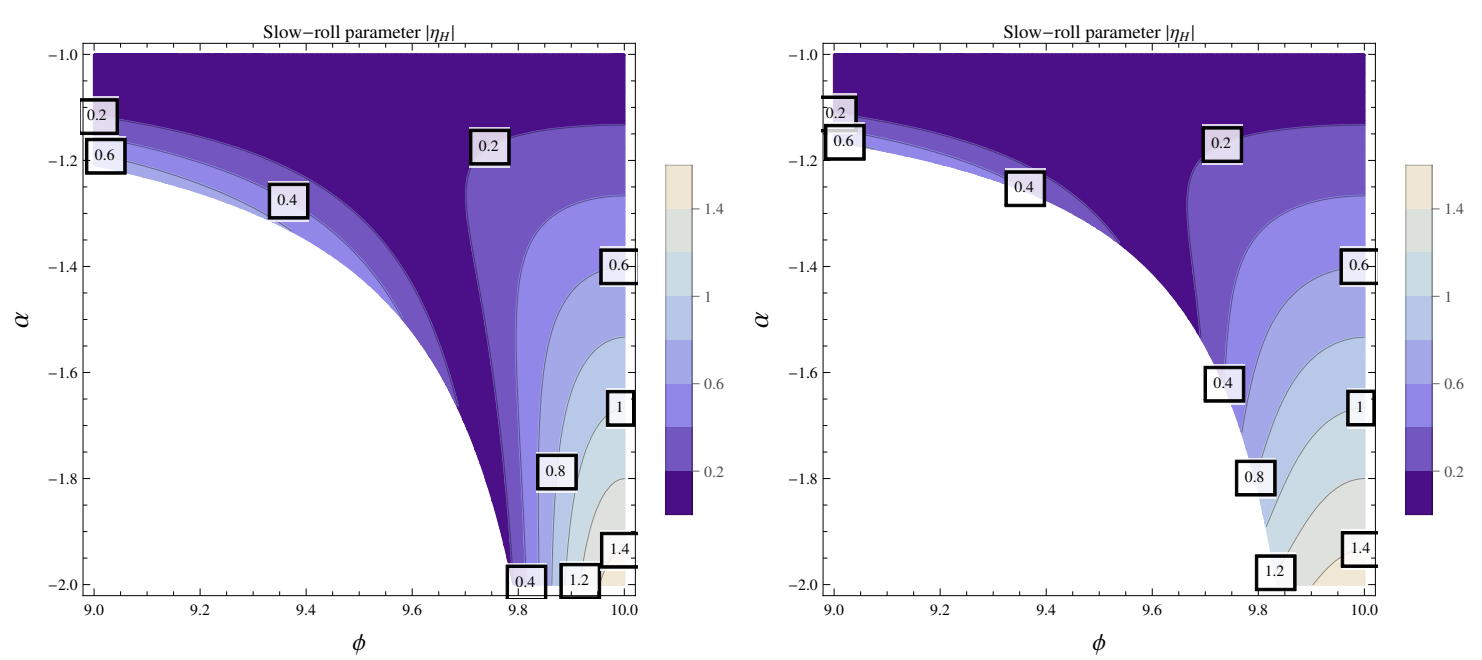

FIG. 9: The contour values of the slow-roll parameter $\eta_{H}$ are plotted as functions of $\phi \leq \phi_{e}(\alpha, \kappa)$ and $-2<\alpha<-1$. On the left (right) we use $\kappa=0.8(0.2)$. Here it can be seen that $\left|\eta_{H}\right|>1$ for $\alpha \leq-5 / 3$.

where $\mathcal{G} \equiv \operatorname{sd}^{2}[F[\arcsin (\sqrt{y(\kappa)}), \kappa]]$. This last equation should be compared with Eq. 32 where $\mathcal{G}$ plays the role of the factor 2 in the denominator. In fact in the limits $\kappa \rightarrow 1$ and $\kappa \rightarrow 0$, we have $\mathcal{G} \rightarrow 2$ and $\mathcal{G} \rightarrow 0.4$ respectively, thus that $\rho_{i}^{1+\alpha} / A$ is now reduced to the boundary $1<\rho_{i}^{1+\alpha} / A<1.4$. This means that the introduction of the new parameter $\kappa$ does not solve the previous problems of $N \ll 60$ for $\alpha<-5 / 3$ since $N_{e}$ does not strongly depend on $\kappa$.

Let us now find an approximation for the values of $\alpha$ such that $\left|\eta_{H}\right|<1$. Similar to Section VI. we expand $\eta_{H}$ in Eq. (52) for $(1+\alpha) \Phi$ small and obtain a similar condition to that in Eq. (34), i.e.

$$
\left|\eta_{H}\right|<1 \quad \text { only for } \quad-\frac{5}{3}<\alpha<-1 \text {. }
$$

In Fig. 9 we plot the contour values of $\eta_{H}$ as function of $\phi$ and $\alpha$. There it can be seen that $\left|\eta_{H}\right|>1$ for all $\alpha \leq-5 / 3$. As we stated before, the introduction of the new parameter $\kappa$ does not 
resolve the slow-roll problem for $\alpha<-5 / 3$. In fact, similar results to the ones in Sec. VI are found for $\xi_{H}, N_{e}, f_{H}$, and the parameters of the model.

\section{CONCLUSIONS}

In this paper we have studied the GCG and GCJG models for primordial inflation in the context of GR. We have shown that the models do not produce inflation when $-1<\alpha<1$, contrary to the results in Refs. [26 28]. Then we show that, although there is a burst of inflation for $\alpha<-1$, the slow-roll parameters $\eta_{H}$ and $\xi_{H}$ are larger than unity for $\alpha<-5 / 3$ and $\alpha<-8 / 5$, respectively. Furthermore, they are much less than unity throughout inflation only for $-1.1<\alpha<-1$. Hence the parameter space of the GCG and GCJG models is greatly reduced.

Then we showed that such a behaviour of the slow-roll parameters implies that the total number of $e$-folds $N$ during inflation is $N \ll 60$ for most of their parameter space, especially for $\alpha<-1.1$. Such that the horizon and flatness problems of the Big Bang cosmology cannot be solved during inflation by the GCG and GCJG models when $\alpha<-1.1$, since the theoretical bound on the number of $e$-folds is $50<N_{*}<60$ [1, 2].

We also constrained the parameters of the models from the Planck 2018 results. From the bounds on the scalar spectral index $n_{s}$ and the tensor-to-scalar ratio $r$, we found that the models are ruled out since they require more than $200 e$-folds before the end of inflation in order to fulfill the bounds.

We conclude that the violation of the slow-roll conditions is a generic feature of the models during inflation when GR is considered and that they are ruled out by the Planck 2018 results.

\section{ACKNOWLEDGEMENTS}

We thank the anonymous referee for valuable remarks and comments which significantly contributed to improve the paper. We also thank Ramón Herrera for useful discussions and Clément Stahl and Gabriel Gómez for useful comments on an early version of the manuscript. A.G.C. was supported by Beca de Inicio Postdoctoral REXE RA N³15/5398/2019 Universidad de Valparaíso.

[1] Planck, Y. Akrami et al., (2018), arXiv:1807.06205.

[2] Planck, Y. Akrami et al., (2018), arXiv:1807.06211. 
[3] A. H. Guth, Phys.Rev. D23, 347 (1981).

[4] A. D. Linde, Phys.Lett. B108, 389 (1982).

[5] A. A. Starobinsky, Grav.Cosmol. 4, 88 (1998), arXiv:astro-ph/9811360.

[6] Martin Jerome et al., Phys. Dark Univ. 5-6, 75 (2014), arXiv:1303.3787.

[7] Y. Wang, Commun. Theor. Phys. 62, 109 (2014), arXiv:1303.1523.

[8] L. Castiblanco, R. Gannouji, and C. Stahl, Large scale structures: from inflation to today: a brief report, 2019, arXiv:1910.03931.

[9] X. Chen, Adv.Astron. 2010, 638979 (2010), arXiv:1002.1416.

[10] S. Chakraborty, T. Paul, and S. SenGupta, Phys. Rev. D98, 083539 (2018), arXiv:1804.03004.

[11] M. C. Bento, O. Bertolami, and A. A. Sen, Phys. Rev. D66, 043507 (2002), arXiv:gr-qc/0202064.

[12] A. A. Sen and R. J. Scherrer, Phys. Rev. D72, 063511 (2005), arXiv:astro-ph/0507717.

[13] B. R. Dinda, S. Kumar, and A. A. Sen, Phys. Rev. D90, 083515 (2014), arXiv:1404.3683.

[14] L. P. Chimento and R. Lazkoz, Class. Quant. Grav. 23, 3195 (2006), arXiv:astro-ph/0505254.

[15] A. Yu. Kamenshchik, U. Moschella, and V. Pasquier, Phys. Lett. B511, 265 (2001), arXiv:grqc/0103004.

[16] J. Lu, Y. Gui, and L. X. Xu, European Physical Journal C 63, 349 (2009), arXiv:1004.3365.

[17] J. C. Fabris, S. V. B. Goncalves, and P. E. de Souza, Gen. Rel. Grav. 34, 53 (2002), arXiv:grqc/0103083.

[18] M. Makler, S. Quinet de Oliveira, and I. Waga, Phys. Lett. B555, 1 (2003), arXiv:astro-ph/0209486.

[19] N. Bilic, G. B. Tupper, and R. D. Viollier, Phys. Lett. B535, 17 (2002), arXiv:astro-ph/0111325.

[20] A. Dev, D. Jain, and J. S. Alcaniz, Phys. Rev. D67, 023515 (2003), arXiv:astro-ph/0209379.

[21] R. Herrera, Phys. Lett. B664, 149 (2008), arXiv:0805.1005.

[22] M. K. Mak and T. Harko, Phys. Rev. D71, 104022 (2005), arXiv:gr-qc/0505034.

[23] A. Jawad, A. Ilyas, and S. Rani, Int. J. Mod. Phys. D26, 1750031 (2016), arXiv:1603.08798.

[24] T. Barreiro and A. A. Sen, Phys. Rev. D70, 124013 (2004), arXiv:astro-ph/0408185.

[25] O. Bertolami and V. Duvvuri, Phys. Lett. B640, 121 (2006), arXiv:astro-ph/0603366.

[26] S. del Campo, JCAP 1311, 004 (2013), arXiv:1310.4988.

[27] J. R. Villanueva, JCAP 1507, 045 (2015), arXiv:1505.03107.

[28] J. R. Villanueva and E. Gallo, Eur. Phys. J. C75, 256 (2015), arXiv:1505.03096.

[29] A. A. Starobinsky, JETP Lett. 55, 489 (1992).

[30] J. A. Adams, B. Cresswell, and R. Easther, Phys. Rev. D64, 123514 (2001), arXiv:astro-ph/0102236.

[31] J. E. Lidsey et al., Rev.Mod.Phys. 69, 373 (1997), arXiv:astro-ph/9508078.

[32] A. R. Liddle and D. H. Lyth, Cosmological inflation and large scale structure (, 2000).

[33] W. H. Kinney, Phys. Rev. D56, 2002 (1997), arXiv:hep-ph/9702427.

[34] A. E. Romano and M. Sasaki, Phys.Rev. D78, 103522 (2008), arXiv:0809.5142.

[35] F. Arroja, A. E. Romano, and M. Sasaki, Phys.Rev. D84, 123503 (2011), arXiv:1106.5384.

[36] P. Adshead, C. Dvorkin, W. Hu, and E. A. Lim, Phys.Rev. D85, 023531 (2012), arXiv:1110.3050. 
[37] X. Chen, R. Easther, and E. A. Lim, JCAP 0706, 023 (2007), arXiv:astro-ph/0611645.

[38] X. Chen, R. Easther, and E. A. Lim, JCAP 0804, 010 (2008), arXiv:0801.3295.

[39] A. G. Cadavid and A. E. Romano, Eur. Phys. J. C75, 589 (2015), arXiv:1404.2985.

[40] A. Gallego Cadavid, J. Phys. Conf. Ser. 831, 012003 (2017), arXiv:1703.04375.

[41] M. Sasaki, Progress of Theoretical Physics 70, 394 (1983). 